\title{
Selección de herbicidas para el control de arvenses en yuca (Manihot esculenta Crantz) en Bolívar, Colombia
}

\author{
Herbicide selection for weed control in cassava \\ (Manihot esculenta Crantz) in Bolivar, Colombia
}
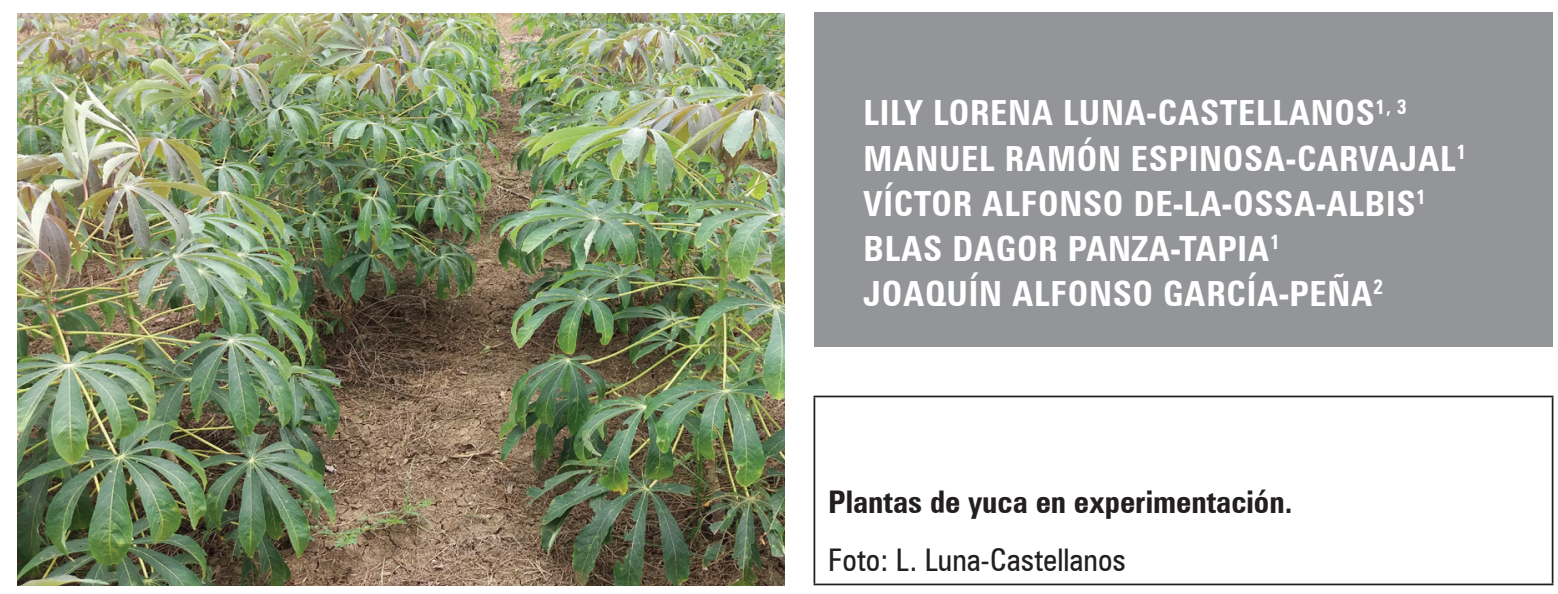

\section{RESUMEN}

La yuca es la raíz tuberosa de mayor importancia para el sustento de la seguridad alimentaria en el departamento de Bolívar (Colombia), sin embargo, el manejo ineficiente de las arvenses durante las primeras etapas de crecimiento ha reducido la productividad, generando pérdidas de rentabilidad para los agricultores de esta región. Por lo tanto, la presente investigación tuvo como objetivo seleccionar herbicidas para el control de arvenses. En la sede Carmen de Bolívar de Corpoica, se implementó un diseño de bloques completamente al azar con tres repeticiones, evaluando las mezclas de herbicidas linuron + pendimetalina, oxadiazón + diuron y metolacloro + atrazina en preemergencia y glufosinato de amonio, haloxifop-p-etoxietil y fluaxifop-p-butil en post-emergencia, sumado a un tratamiento de control. Las variables evaluadas, eficiencia en el control de arvenses, sobrevivencia, grado de fitotoxicidad, altura, diámetro del tallo y rendimiento de raíces frescas en plantas de yuca, mostraron que la mezcla de metolacloro + atrazina presentaba la mayor eficiencia en el control de arvenses y selectividad al cultivo de yuca, aplicada en preemergencia, obteniendo un incremento del rendimiento en 4,34 tha $\mathrm{h}^{-1}$ en comparación con el tratamiento control manual. Los resultados indican la efectividad de los herbicidas dentro del manejo integrado de arvenses en cultivos de yuca en la Costa Caribe colombiana.

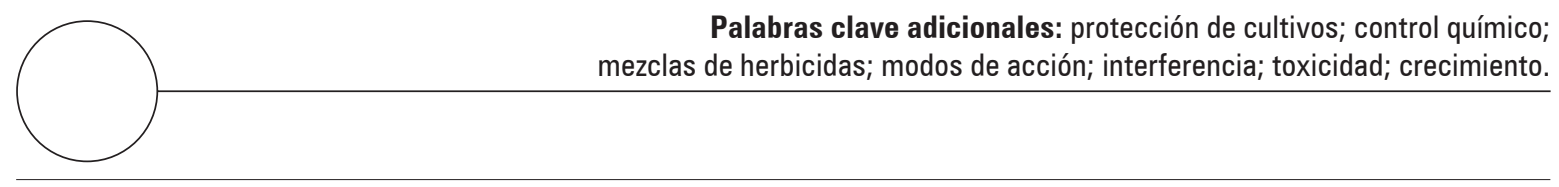

1 Corporación Colombiana de Investigación Agropecuaria (AGROSAVIA), Centro de Investigación Turipana, Carmen de Bolívar (Colombia). ORCID Luna-Castellanos, L.L.: 0000-0003-2172-7842; ORCID Espinosa-Carvajal, M.R.: 00000001-8939-5472; ORCID De-La-Ossa-Albis, V.A.: 0000-0003-0038-3008; ORCID Panza-Tapia, B.D.: 0000-0002-90413620

2 Corporación Colombiana de Investigación Agropecuaria (AGROSAVIA), Centro de Investigación Turipana, Cerete (Colombia). ORCID García-Peña, J.A.: 0000-0002-1805-9487

3 Autor para correspondencia.1lunac@agrosavia.co 


\section{ABSTRACT}

Cassava is the most important tuberous root for food security in the Department of Bolívar (Colombia); however, inefficient weed management during early growth stages has reduced productivity, generating profit losses for farmers in this region. Therefore, this research aimed to select herbicides for weed control. At Carmen de Bolívar de Corpoica, a completely randomized block design was used with three repetitions, evaluating the herbicide mixtures linuron + pendimethalin, oxadiazon + diuron and metolachlor + atrazine in pre-emergence, and glufosinate ammonium, haloxyfop-p-ethoxyethyl and fluaxifop-p-butyl in post-emergence, along with a control treatment. The evaluated variables weed control efficiency, survival, degree of phytotoxicity, height, stem diameter and yield of fresh roots in cassava plants showed that the mixture metolachlor + atrazine applied in pre-emergence had the highest efficiency for weed control and cassava crop selectivity, obtaining a yield increase of $4.34 \mathrm{tha} \mathrm{h}^{-1}$, as compared to the manual control treatment. The results indicated the effectiveness of herbicides within integrated weed management in cassava crops on the Colombian Caribbean Coast.

Additional key words: crop protection; chemical control; herbicide mixtures; mode of action; interference; toxicity; growth.

Fecha de recepción: 21-03-2018 Aprobado para publicación: 30-11-2018

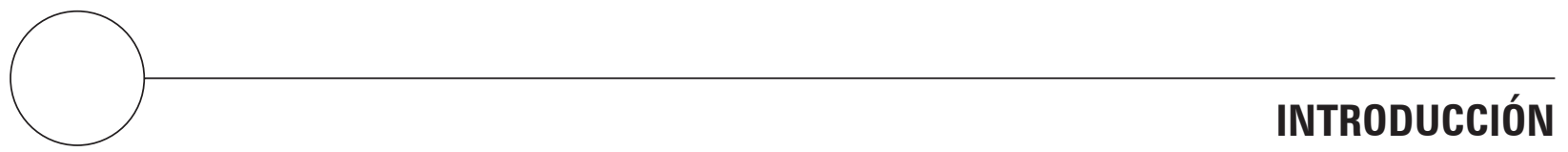

La yuca (Manihot esculenta Crantz) es un cultivo de importancia en las regiones tropicales y subtropicales del mundo, debido a que representa una buena fuente de energía (Owusu-Darko et al., 2014; Villordon et al., 2014). Su contribución al bienestar y seguridad alimentaria de la población y versatilidad de los sistemas productivos le confieren importancia a este cultivo para los agricultores con recursos limitados (Reddy, 2015; Nduwumuremyi et al., 2017).

Las raíces de la yuca y los subproductos obtenidos a partir de esta planta representan gran valor económico para el desarrollo agrícola a nivel global. Según la FAO (2016), la producción mundial fue de 277,102 millones de toneladas, que se cultivaron en 23,482 millones de hectáreas, de este volumen $30-60 \%$ es producido en África, $30-40 \%$ en Asia y $15-20 \%$ en América latina. Colombia con un área plantada de 210.250 ha, volumen de producción de 2,105.436 millones de toneladas y rendimiento promedio de 10,01 $t$ ha ${ }^{-1}$ es el tercer productor de yuca en Latinoamérica después de Brasil y Paraguay (Pérez et al., 2018). El sistema productivo de yuca en Colombia se concentra en la región Caribe, la cual representa el 65\% del total del área sembraba a nivel nacional, según AGRONET (2016) los mayores rendimientos $11,61 \mathrm{t} \mathrm{ha}^{-1}$ son obtenidos en el departamento de Córdoba, seguido de Atlántico $\left(10,34\right.$ t ha $\left.{ }^{-1}\right)$, Bolívar $\left(9,66\right.$ t ha $\left.{ }^{-1}\right)$, Magdalena $\left(7,61 \mathrm{t} \mathrm{ha}^{-1}\right)$ y Sucre $\left(7,77 \mathrm{t} \mathrm{ha}^{-1}\right)$, respectivamente.
La yuca constituye un reglón importante para el sustento de la seguridad alimentaria en el departamento de Bolívar, ocupa el segundo lugar en superficie sembrada (47.290 ha) después del maíz tradicional (AGRONET, 2016). Los agricultores de Bolívar, en su mayoría corresponden a pequeños agricultores, los cuales desconocen las prácticas agronómicas adecuadas de manejo de estacas (cangres), suelos, arvenses y enfermedades para aumentar la productividad del cultivo (Aguilera, 2012). Estudios realizados por Arrieta et al. (2004), revelaron que el control ineficiente de las poblaciones de arvenses asociadas a los cultivos de yuca genera pérdidas en rendimiento del $50 \%$ y eleva los costos de producción en $40 \%$ debido a la excesiva utilización de mano de obra requerida para ejecutar el control manual.

Las pérdidas en rendimiento en la yuca debido a arvenses no controladas durante el periodo crítico pueden alcanzar pérdidas hasta en un $75-100 \%$ (Silva et al., 2013). La yuca es un cultivo de larga duración (8-12 meses), con un crecimiento y una tasa de desarrollo de la parte aérea en sus fases iniciales de crecimiento muy lento (Moura, 2000; Johanns y Contiero, 2006). Por lo general, se cultiva en hileras espaciadas a $1,0 \times 1,5 \mathrm{~m}$, lo que permite que las arvenses se desarrollen rápidamente (Nedunchezhiyan et al., 2013; Streck et al., 2014; Salau et al., 2015). Las arvenses también afectan la calidad de las raíces de 
yuca al competir de forma eficiente por los recursos ambientales disponibles. Por lo tanto, el control de las arvenses es crítico, particularmente en los primeros 3-4 meses de crecimiento de la yuca (Nedunchezhiyan et al., 2017; Weerarathne et al., 2017). El control de arvenses en yuca se logra a través del desyerbe manual u otros métodos culturales, mientras que, en las áreas con escasez de mano de obra, se practica el control químico de arvenses (Vencill et al., 2012). Sin embargo, la utilización de herbicidas es baja en la mayoría de las regiones de cultivo de yuca del mundo, debido a la falta de disponibilidad de herbicidas específicamente desarrollados para la yuca y su baja persistencia (Silveira et al., 2012; Enyong et al., 2013).

La implementación del método químico para el control de arvenses en yuca requiere de una adecuada selección del herbicida. En el mundo existen 12 herbicidas registrados para uso en el cultivo de yuca, se encuentran agrupados en seis mecanismos de acción (Varanasi et al., 2016; Zimdahl, 2018): 1) inhibidores de la fotosíntesis en el fotosistema II (diuron, linuron, atrazina), que inducen cambios que conllevan a la destrucción de la clorofila, impidiendo la absorción de $\mathrm{CO}_{2}$ y la reacción de Hill, provocan colapso de las hojas en plántulas jóvenes; 2 ) inhibidores de la oxidasa del fotoporfirinogeno (PPO) (oxadiazon, oxifluorfen), que conlleva a la formación de radicales libres que alteran la formación de precursores de clorofila e inducen la desintegración de la membrana celular, que facilita la deshidratación y desintegración rápida de las células; 3 ) inhibidores de la polimerización de la tubulina del ensamblaje de microtúbulos (pendimetalina), que son inhibidores del crecimiento, más específicamente de la elongación de las raíces, lo que provoca la muerte rápida de la maleza. Inhibidores de la división celular (alaclor, butaclor, metolacloro) que afectan el metabolismo de lípidos, la síntesis de proteínas y la formación de ceras en la cutícula; 4) inhibidores de la síntesis de la glutamina (glufosinato de amonio), que reduce la fuente de amonio disponible para enzimas e inhibe la fotorrespiración, y finalmente los 5) inhibidores del acetil Coenzima A carboxilasa (ACCase) (fluazifop-p-butil, haloxyfop-p-etoxietil), que afectan la síntesis de lípidos deteriorando rápidamente los tejidos jóvenes en crecimiento.

La respuesta de la yuca a la aplicación de herbicidas depende de la selectividad del herbicida, modo y mecanismo de acción, época de aplicación, tipo de suelo, condiciones climáticas, variedad de yuca y tipo de arvenses. Teniendo en cuenta que las investigaciones en torno a la evaluación de herbicidas en el sistema productivo de yuca en el departamento de Bolívar son limitadas, la presente investigación tuvo como objetivo realizar la selección de herbicidas para el control de arvenses en yuca (M. esculenta) en Bolívar, Colombia. Las mejores moléculas podrán ser utilizadas por los agricultores, como una alternativa de manejo eficiente que ayudará a mejorar la productividad de esta raíz tuberosa.

\section{MATERIALES Y MÉTODOS}

El experimento se llevó a cabo en la granja experimental de AGROSAVIA sede Carmen de Bolívar (Bolívar, Colombia), coordenadas $9^{\circ} 42,50,8^{\prime \prime} \mathrm{N}$ y $75^{\circ} 06^{\prime} 26,9^{\prime \prime}$ $\mathrm{W}$, temperatura promedio de $27,7^{\circ} \mathrm{C}$, humedad relativa promedio de $76 \%$, altitud de $143 \mathrm{msnm}$, régimen bimodal de lluvias con precipitación media de 1100 $\mathrm{mm}$ anuales. El área pertenece a la zona climática cálido muy seco, a la formación de bosque seco tropical (BS-T) (Guevara et al., 2004). El suelo donde se instaló el experimento presentó textura franco-arcillosa, $\mathrm{pH}$ 7,57; MO 2,01\%; P 55,28 mg kg-1; $\mathrm{Ca}^{2+} 26,67 \mathrm{cmol} \mathrm{kg}$ ${ }^{1}, \mathrm{Mg}^{2+} 5,66 \mathrm{cmol} \mathrm{kg}^{-1}, \mathrm{~K} 1,00 \mathrm{cmol} \mathrm{kg}{ }^{-1}$ y CIC $=34,36$ $\mathrm{cmol} \mathrm{kg}$.

Las estacas o cangres de la variedad MCOL-2215 'venezolana' de longitud $20 \mathrm{~cm}$, diámetro $3 \mathrm{~cm}$ y entre 5-6 yemas, fueron obtenidos del tercio medio de plantas maduras de yuca (10-12 meses de edad) en buen estado fitosanitario. La desinfección se efectuó sumergiendo los cangres durante 5 min en una mezcla a base de fungicida (metalaxil + mancozeb: $3 \mathrm{~g} \mathrm{~L}^{-1}$ ) e insecticida (fipronil: $3 \mathrm{cc} \mathrm{L}^{-1}$ ).

El estudio se desarrolló en la temporada comprendida entre agosto de 2016 y agosto de 2017. El suelo se preparó de forma mecánica realizando dos pases de rastra liviana. Las arvenses emergentes fueron controladas mediante la aplicación del herbicida glifosato (concentración: $480 \mathrm{~g} \mathrm{~L}^{-1}$ ) en dosis de 12,5 cc L-1 $8 \mathrm{~d}$ antes de la siembra.

Se utilizó un diseño de bloques completos al azar con siete tratamientos y tres repeticiones. La unidad experimental (UE) consistió en cinco hileras de $7 \mathrm{~m}$ espaciadas a $1 \mathrm{~m}$ y $0,8 \mathrm{~m}$ entre plantas para un área total de $35 \mathrm{~m}^{2}$. El área útil fue $9,6 \mathrm{~m}^{2}$ correspondiente a 3 hileras centrales descartando las plantas de los extremos. 


\section{Herbicidas y épocas de aplicación}

Diferentes combinaciones de ingredientes activos y desyerbes manuales fueron implementados como tratamientos (Tab. 1). Las mezclas fueron realizadas teniendo en cuenta el grado de compatibilidad, mecanismo de acción y época de aplicación. La aplicación de herbicidas fueron realizadas en dos épocas del cultivo. En preemergencia se aplicaron las siguientes combinaciones: linuron + pendimentalina; oxadiazón + diuron y metolacloro + atrazina. En post-emergencia posterior a la brotación de los cangres y cuando se presentó la emergencia de arvenses: fluazifop, haloxifop y glufosinato. Se empleó una aspersora o pulverizador manual con capacidad de $20 \mathrm{~L}$ y boquilla TK 2.5.

Se evaluaron las siguientes variables:

Porcentaje de sobrevivencia (\%): se realizó la evaluación de sobrevivencia de arvenses trascurridos 30 días después de la siembra (DDS) y aplicados los herbicidas de pre-emergencia. Para los tratamientos en post-emergencia se determinó a los 10 días después de la aplicación (DDA).

Crecimiento del cultivo: se registró la altura $(\mathrm{cm})$ de 5 plantas a los 30, 45, 60 y 75 DDS y al momento de la cosecha. Para el diámetro ( $\mathrm{mm})$ a los 12 meses de sembrado el cultivo.

Evaluación de fitotoxicidad: la fitotoxicidad de los herbicidas sobre las plantas de yuca se valoró a través de la escala visual EWRC adaptada de Scariot et al. (2013) donde 1 corresponde a planta sana, 2 clorosis leve, 3 amarillamiento generalizado con escaso desarrollo de hojas y tallo, 4 clorosis, quemazón en bordes de hojas y encrespamiento, y 5 planta muerta.
Las evaluaciones fueron realizadas al momento de la brotación entre los 15, 30, 45 y 60 DDA de los herbicidas pre-emergentes. En el caso de los herbicidas post-emergentes $1,7,15$ y 30 DDA seleccionando 6 plantas al azar.

Caracterización de la comunidad de arvenses y eficiencia de los herbicidas en el Control: se utilizó el método de la cuadricula $\left(25 \mathrm{~m}^{2}, 5 \times 5 \mathrm{~m}\right)$ para determinar el porcentaje de infestación a los 50 DDS. Se lanzó una vez al azar sobre el área efectiva. Las arvenses fueron identificadas según la familia, género y especie. Se implementó la escala visual de Alemán (2004) (Tab. 2) para determinar el porcentaje de control que ejerce la aplicación de herbicidas sobre las arvenses.

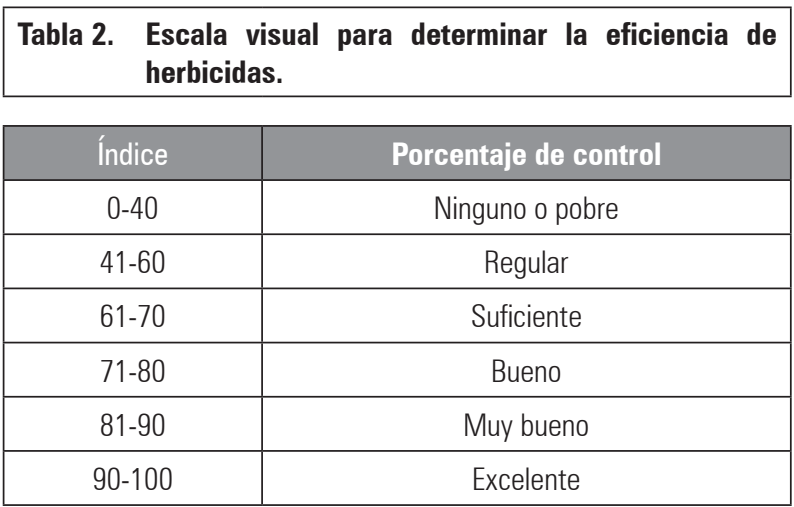

Fuente: Alemán (2004)

Rendimiento de raíces frescas $\left(t h a^{-1}\right)$ : La cosecha se realizó de forma manual a los 12 meses de edad. Se determinaron los componentes de rendimiento número y peso $(\mathrm{kg})$ de raíces comerciales y no comerciales.

Se realizaron análisis de normalidad y homogeneidad de varianzas mediante las pruebas de Shapiro-Wilk

Tabla 1. Tratamientos evaluados para el control de arvenses en época de preemergencia y post-emergencia en el cultivo de yuca.

\begin{tabular}{|l|l|c|c|}
\hline \multicolumn{1}{|c|}{ Tratamientos } & \multicolumn{1}{|c|}{ Descripción } & Época de aplicación & Dosis ia (ha) \\
\hline Linuron + pendimentalina & Linuron + pendimentalina + control manual en postemergencia & Preemergencia & $1,5 \mathrm{~kg}+3,5 \mathrm{~L}$ \\
\hline Oxadiazón + diuron & Oxadiazón + diuron + control manual en postemergencia & Preemergencia & $2,0 \mathrm{~L}+1,5 \mathrm{~L}$ \\
\hline Metolacloro + atrazina & Metolacloro + atrazina + control manual en postemergencia, & Preemergencia & $1,5 \mathrm{~L}+2,0 \mathrm{~kg}$ \\
\hline Fluazifop & Fluazifop-p-butil + control manual & Post-emergente & $2,0 \mathrm{~L}$ \\
\hline Haloxifop & Haloxifop-R-metil ester + control manual & Post-emergente & $400 \mathrm{~cm}^{3}$ \\
\hline Glufosinato & Glufosinato de amonio + control manual & Post-emergente & $2,0 \mathrm{~L}$ \\
\hline Control manual & Testigo: control manual & - & - \\
\hline
\end{tabular}

ia, ingrediente activo 
y Levene, respectivamente. Comprobados los supuestos, se realizó un análisis de varianza y pruebas de comparación múltiple de Tukey al 5\%. Se empleó el paquete estadístico SAS v. 9.2.

\section{RESULTADOS Y DISCUSIÓN}

El análisis de varianza no reveló diferencias estadísticas significativas $(P>0,05)$ en la sobrevivencia de plantas de yuca (Tab. 3). Resultados similares fueron reportados por Matheus et al. (2004), quienes evaluaron el efecto de tres herbicidas (fluometurón, metolacloro y pendimetalína) durante el establecimiento en campo de plántulas propagadas in vitro que no interrumpieron los procesos fisiológicos de crecimiento. Costa et al. (2013) determinaron que los herbicidas pre-emergentes, linuron, oxadiazon, y alaclor, son moderadamente selectivos al cultivo de yuca, ya que no generan daños a la planta siempre y cuando se utilicen en dosis recomendadas para cada tipo de suelo y aplicados en un periodo no mayor a 5 DDS.

La combinación de metolacloro + atrazina afectó en menor medida la sobrevivencia de yuca (Tab. 4). Al respecto, Biffe et al. (2010) determinaron que la aplicación de estos herbicidas en esta etapa del cultivo proporciona control satisfactorio de las arvenses y no genera daños en la morfología inicial del crecimiento, por lo que constituye en la opción viable en los

Tabla 3. Análisis de varianza de sobrevivencia, diámetro de tallo y rendimiento en plantas de yuca implementando diferentes herbicidas para mejorar la eficiencia en el control de arvenses.

\begin{tabular}{|c|c|c|c|c|c|}
\hline \multicolumn{6}{|c|}{ Sobrevivencia (\%) } \\
\hline FV & $\mathrm{GL}$ & SC & $\mathrm{CM}$ & F-valor & $\operatorname{Pr}>\mathrm{F}$ \\
\hline Bloques & 2 & 19,3936 & 9,6968 & 0,87 & 0,4419 NS \\
\hline Tratamiento & 6 & 42,0257 & 7,0042 & 0,63 & $0,7030^{N S}$ \\
\hline Error & 12 & 133,0064 & & & \\
\hline Total & 20 & 194,4789 & & & \\
\hline
\end{tabular}

\begin{tabular}{|c|c|c|c|c|c|}
\hline \multicolumn{6}{|c|}{ Diámetro del tallo (mm) } \\
\hline Bloques & 2 & 57,7188 & 28,8594 & 2,75 & 0,1038 NS \\
\hline Tratamiento & 6 & 73,4311 & 12,2385 & 1,17 & $0,3845^{N S}$ \\
\hline Error & 12 & 125,8445 & & & \\
\hline Total & 20 & & & & \\
\hline
\end{tabular}

\begin{tabular}{|l|c|c|c|c|c|}
\hline \multicolumn{7}{|c|}{ Eficiencia del control de arvenses (\%) } & 1,89 & 0,1935 \\
\hline Bratamiento & 2 & 5,42857 & 2,71429 & 2523,01 & $<0,0001^{* *}$ \\
\hline Error & 6 & 21745,90 & 3624,31 & & \\
\hline Total & 12 & 17,23810 & 1,43651 & & \\
\hline
\end{tabular}

CV $(\%)=2,55$

\begin{tabular}{|l|c|c|c|c|c|}
\hline \multicolumn{5}{|c|}{ Rendimiento de raíces (t ha- $\left.{ }^{-1}\right)$} \\
\hline Bloques & 2 & 9,2181 & 4,6090 & 3,90 & $0,0497^{*}$ \\
\hline Tratamiento & 6 & 95,4983 & 15,9163 & 13,46 & $0,0001^{* *}$ \\
\hline Error & 12 & 14,1932 & & & \\
\hline Total & 20 & 118,9098 & & & \\
\hline CV $(\%)=8,86$ &
\end{tabular}

GL: grados de libertad; SC: suma de cuadrados; CM: cuadrados medios; CV: coeficiente de variación; ${ }^{* *}=$ significativo 1\%; " $=$ significativo al $5 \%$; ${ }^{\text {ss: }}$ sin diferencias significativa. 
programas de manejo integrado de arvenses en el cultivo de yuca. El herbicida glufosinato de amonio en post-emergencia presento la sobrevivencia de plantas de yuca en un 98,09\% (Tab. 4). Estos resultados contrastan con los reportados por Silva et al. (2010), quienes encontraron que este herbicida redujo en $40 \%$ la sobrevivencia de plantas de yuca, en las cuales se observó una alta toxicidad hasta los $35 \mathrm{DDA}$, las plantas presentaron clorosis generaliza, quemazón de hojas y las raíces tuberosas cosechadas presentaron menor porcentaje de materia seca.

Los herbicidas evaluados no ejercieron efectos significativos $(P>0,05)$ sobre el diámetro del tallo (Tab. 3 y 4) y la altura de plantas de yuca (Fig. 1).

La yuca es una planta resistente a la aplicación de varios grupos de herbicidas, sin embargo, cada variedad presenta una respuesta diferencial en su crecimiento dependiendo de las condiciones ambientales, el mecanismo de acción del herbicida y la época de aplicación (Silva et al., 2011).

Estudios realizados por Gutiérrez et al. (2008) y Silva et al. (2012), comprobaron que los herbicidas inhibidores del fotosistema II (diuron, atrazina, ametrina, linuron) aplicados 60 DDS en el cultivar IAC-12 bajo condiciones controladas en un suelo de textura arcillosa, no manifestaron consecuencias sobre el área foliar, número de hojas, altura de planta y diámetro del tallo en comparación con las plantas no tratadas con herbicidas. De manera similar Da Costa et al. (2014), reportaron que los herbicidas aplicados en época de preemergencia (clomazone, metsulfuron-methyl, y carfentrazone), solos y en mezcla con glifosato, sobre el cultivar Fecula blanca, no afectaron de manera negativa el crecimiento de las plantas de yuca.

Tabla 4. Efecto de herbicidas aplicados en época de preemergencia y post-emergencia sobre la sobrevivencia de plantas (\%), diámetro de tallo, eficiencia en el control de arvenses (\%), rendimiento de raíces de yuca.

\begin{tabular}{|l|c|c|c|c|}
\hline \multicolumn{1}{|c|}{ Tratamiento } & Sobrevivencia (\%) & Diámetro tallo & $\begin{array}{c}\text { Eficiencia en el control } \\
\text { de arvenses (\%) }\end{array}$ & $\begin{array}{c}\text { Rendimiento de raíces } \\
\left(\mathrm{t} \text { ha }{ }^{-1} \text { ) }\right.\end{array}$ \\
\hline Linuron + pendimentalina & $95,24 \pm 2,77 \mathrm{a}$ & $27,50 \pm 2,24 \mathrm{a}$ & $66,0 \pm 0,28 \mathrm{~b}$ & $13,63 \pm 0,62 \mathrm{abc}$ \\
\hline Oxadiazón + diuron & $95,23 \pm 2,32 \mathrm{a}$ & $26,13 \pm 2,82 \mathrm{a}$ & $68,3 \pm 0,84 \mathrm{~b}$ & $15,23 \pm 0,92 \mathrm{a}$ \\
\hline Metolacloro + atrazina & $98,10 \pm 1,22 \mathrm{a}$ & $29,76 \pm 1,57 \mathrm{a}$ & $79,7 \pm 0,61 \mathrm{a}$ & $14,74 \pm 0,08 \mathrm{ab}$ \\
\hline Fluazifop & $95,24 \pm 0,85 \mathrm{a}$ & $26,50 \pm 0,54 \mathrm{a}$ & $0,0 \pm 0,35 \mathrm{~d}$ & $9,30 \pm 0,37 \mathrm{~d}$ \\
\hline Haloxifop & $98,09 \pm 1,63 \mathrm{a}$ & $26,73 \pm 1,32 \mathrm{a}$ & $0,0 \pm 0,35 \mathrm{~d}$ & $10,67 \pm 1,10 \mathrm{~cd}$ \\
\hline Glufosinato & $98,09 \pm 1,63 \mathrm{a}$ & $23,82 \pm 0,61 \mathrm{a}$ & $76,3 \pm 0,31 \mathrm{a}$ & $11,83 \pm 0,52 \mathrm{bdc}$ \\
\hline Control manual & $95,24 \pm 1,22 \mathrm{a}$ & $29,41 \pm 1,73 \mathrm{a}$ & $37,7 \pm 1,15 \mathrm{c}$ & $10,40 \pm 1,00 \mathrm{~d}$ \\
\hline
\end{tabular}

Promedios con letras distintas en la columna, indican diferencia significativa entre tratamientos de acuerdo con la prueba Tukey $(P \leq 0,05)$.

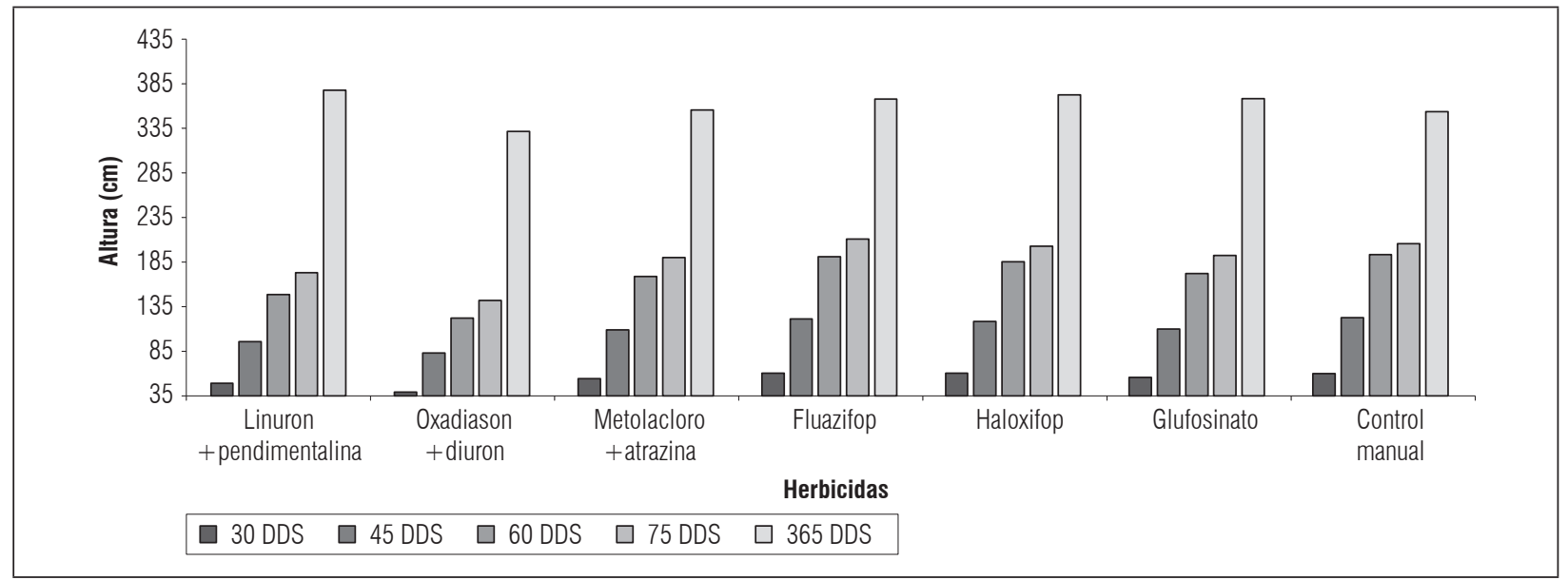

Figura 1. Efecto de herbicidas sobre la altura de plantas de yuca. 
No se encontraron diferencias estadísticas significativas entre los tratamientos para la variable altura de la planta. Las plantas tratadas con la mezcla oxadiazón + diuron presentaron menor altura $(121,4 \mathrm{~cm})$ en comparación con las plantas testigo (control manual, 192,1 cm) (Fig. 1). Los herbicidas inhibidores de la oxidasa del fotoporfirinogeno (PPO) como oxadiazón desestabilizan la formación de precursores de la clorofila, inhiben la protoporfirinogeno-9-oxidasa que participa en la captura de luz, lo que conlleva a la reducción de la actividad fotosintética de la planta y por consiguiente disminuye la producción de biomasa (Alves et al., 2015).

\section{Fitotoxicidad de los herbicidas}

Los herbicidas aplicados en preemergencia causan síntomas visibles de toxicidad en la parte aérea de la planta (Tab. 5), dichos tratamientos, linuron + pendimentalina, oxadiazón + diuron y metolacloro + atrazina, presentaron al momento de la brotación y trascurridos 15 DDA, una ligera clorosis, quemazón de hojas y un encrespamiento de los ápices, las plantas muestran recuperación completa del follaje para linuron + pendimentalina y oxadiazón + diuron a los $60 \mathrm{DDA}$, mientras que metolacloro + atrazina síntomas se observan a los 23 DDA. Estos resultados son similares a los reportados por Biffe et al. (2010), quienes indicaron que las variedades Fécula blanca y Fibra los síntomas de toxicidad no fueron visibles sino a los 30 DDA. Trabajos realizados por Franciscon et al. (2016) sobre eficacia y selectividad de mezclas de herbicidas en yuca, determinaron que los herbicidas a base de metolacloro presentan fitotoxicidad media al cultivo, alcanzando una recuperación a los 70 DDA.
Los tratamientos fluazifop, haloxifop y glufosinato aplicados con pantalla y de forma dirigida no ejercieron efectos notorios de fitotoxicidad sobre las plantas de yuca. De acuerdo con Costa et al. (2013), el herbicida fluazifop-p-butil no genera toxicidad en las plantas cuando es aplicado de forma dirigida, las plantas no expresan síntomas visibles de afectación por el herbicida.

\section{Caracterización de la comunidad arvense y eficiencia de herbicidas}

A los 50 DDS y después de ser aplicados los herbicidas la comunidad arvense estuvo compuesta por cuatro familias y cuatro especies (Tab. 6). La especie de mayor frecuencia correspondió a Cyperus rotundus L., seguida de Kallstroemia maxima (L.) Hook. \& Arn., Cassia tora L. y Amaranthus dubius Mart. ex Thell., respectivamente (Fig. 2). Las especies identificadas en este estudio también han sido reportadas en yuca (Olorunmaiye et al., 2013) y cultivos de batata (Lugo-Torres y Diaz, 2007). El éxito de C. rotundus presente en todos los tratamientos se explica a la eficiente absorción de $\mathrm{CO}_{2}$ atmosférico y la liberación de sustancias alelopáticas perjudiciales a otras especies arvenses y cultivos. Además, la reproducción a través de rizomas y tubérculos le confieren la capacidad de multiplicarse rápidamente en periodos muy cortos, sobrevivir por años a profundidades de $20 \mathrm{~cm}$ en el suelo y lograr procesos de dispersión exitosos que conllevan a la invasión y colonización eficiente de áreas de cultivos (Aramendiz et al., 2010).

La eficiencia de las mezclas de herbicidas en el control de arvenses registró diferencias significativas (Tab. 3), lo cual indica que hubo un efecto notorio de algunos

Tabla 5. Fitotoxicidad de herbicidas con la escala visual EWRC en yuca.

\begin{tabular}{|c|c|c|c|c|c|c|}
\hline \multirow{2}{*}{ Tratamientos } & \multicolumn{6}{|c|}{ Fitotoxicidad } \\
\hline & Brotación & 15 DDS & $23 \mathrm{DDS}$ & 30DDS & 45DDS & 60DDS \\
\hline Linuron + pendimentalina & 4,0 & 4,0 & 3,0 & 2,0 & 2,0 & 1,0 \\
\hline Oxadiazón + diuron & 4,0 & 4,0 & 3,0 & 2,0 & 2,0 & 1,0 \\
\hline Metolacloro + atrazina & 2,0 & 2,0 & 1,0 & 1,0 & 1,0 & 1,0 \\
\hline Fluazifop & 1,0 & 1,0 & 1,0 & 1,0 & 1,0 & 1,0 \\
\hline Haloxifop & 1,0 & 1,0 & 1,0 & 1,0 & 1,0 & 1,0 \\
\hline Glufosinato & 1,0 & 1,0 & 1,0 & 1,0 & 1,0 & 1,0 \\
\hline Control manual & 1,0 & 1,0 & 1,0 & 1,0 & 1,0 & 1,0 \\
\hline
\end{tabular}

DDS: días después de la siembra. 
Tabla 6. Arvenses identificadas posterior a la aplicación de herbicidas pre-emergentes y post-emergentes en el cultivo de yuca en la Costa Caribe colombiana.

\begin{tabular}{|c|c|c|c|c|c|}
\hline Tratamiento & Familia & Género & Especie & Nombre vulgar & \% Infestación \\
\hline Linuron + pendimentalina & Cyperaceae & Cyperus & C. rotundus & Coquito & 40 \\
\hline Oxadiazón + diuron & Cyperaceae & Cyperus & C. rotundus & Coquito & 25 \\
\hline Metolacloro + atrazina & Cyperaceae & Cyperus & C. rotundus & Coquito & 15 \\
\hline \multirow{3}{*}{ Fluazifop } & Cyperaceae & Cyperus & C. rotundus & Coquito & 10 \\
\hline & Caesalpiniaceae & Cassia & C. tora & Bicho & 15 \\
\hline & Zygophyllaceae & Kallstroemia & K. maxima & Atarraya & 75 \\
\hline \multirow{4}{*}{ Haloxifop } & Cyperaceae & Cyperus & C. rotundus & Coquito & 10 \\
\hline & Amaranthaceae & Amaranthus & A. dubius & Bledo blanco & 35 \\
\hline & Caesalpiniaceae & Cassia & C. tora & Bicho & 15 \\
\hline & Zygophyllaceae & Kallstroemia & K. máxima & Atarraya & 40 \\
\hline \multirow{4}{*}{ Glufosinato } & Cyperaceae & Cyperus & C. rotundus & Coquito & 4 \\
\hline & Amaranthaceae & Amaranthus & A. dubius & Bledo blanco & 6 \\
\hline & Caesalpiniaceae & Cassia & C. tora & Bicho & 5 \\
\hline & Zygophyllaceae & Kallstroemia & K. maxima & Atarraya & 7 \\
\hline \multirow{4}{*}{ Control manual } & Cyperaceae & Cyperus & C. rotundus & Coquito & 10 \\
\hline & Amaranthaceae & Amaranthus & A. dubius & Bledo blanco & 35 \\
\hline & Caesalpiniaceae & Cassia & C. tora & Bicho & 15 \\
\hline & Zygophyllaceae & Kallstroemia & K. maxima & Atarraya & 40 \\
\hline
\end{tabular}

herbicidas sobre la disminución en el porcentaje de infestación de arvenses. La mezcla metolacloro + atrazina controló $79,7 \%$ la emergencia de arvenses, este tratamiento mostró el mayor grado de eficiencia de control en preemergencia (Tab. 4). Scariot et al. (2013) corroboraron que metolacloro aplicado solo

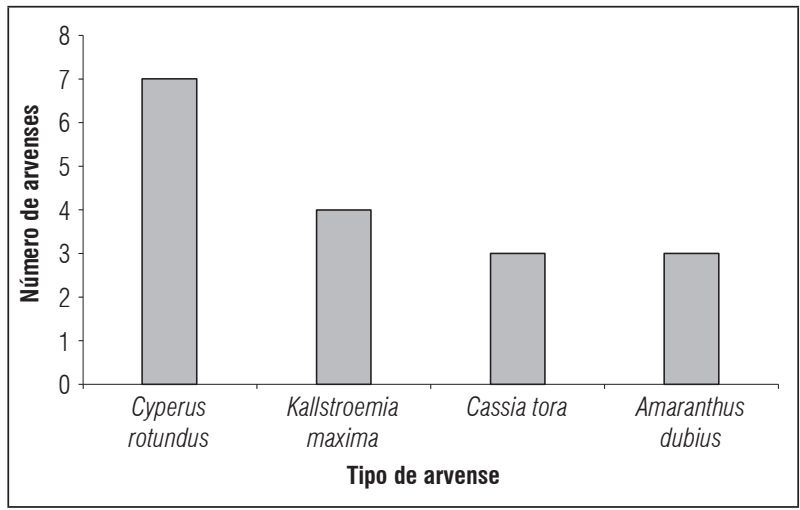

Figura 2. Frecuencia absoluta de arvenses asociadas al cultivo de yuca después de la aplicación de diferentes herbicidas en la Costa Caribe colombiana. o en mezcla con clomazone proporciona un control excelente de arvenses hasta los 105 DDA en yuca 'Cascuda'. Para el caso de los tratamientos linuron + pendimentalina y oxadiazón + diuron, se encontró un control suficiente para las arvenses.

El tratamiento glufosinato aplicado en post-emergencia ejerció un control del 76,3\% de las arvenses, catalogándose bueno según la escala propuesta por Alemán (2004). Los herbicidas fluazifop y haloxifop, obtuvieron un control pobre ( $0 \%)$ sobre las comunidades de arvenses de hoja ancha y C. rotundus, este hecho se fundamenta en la especificidad de las moléculas para el control de las gramíneas.

El control manual es la práctica común de las plantaciones de yuca, este método ejerció un control regular $(37,7 \%)$ de las arvenses, mostrando poca eficiencia en el control, por lo cual, las arvenses colonizan rápidamente la superficie del suelo compitiendo de forma directa por agua, luz y nutrientes con las plantas de yuca, incrementando el número de controles y por consiguiente los costos de producción del cultivo (Weerarathne et al., 2017). 


\section{Rendimiento de raíces tuberosas}

El efecto de las mezclas de herbicidas sobre el rendimiento de raíces tuberosas de yuca presentó diferencias significativas $(P \leq 0,01)$ (Tab. 3$)$. Las aplicaciones en preemergencia, linuron + pendimentalina, oxadiazón + diuron y metolacloro + atrazina, mostraron incrementos sustanciales en el rendimiento en comparación al tratamiento control manual (Tab. 4). Estas mezclas presentaron baja fitotoxicidad en las plantas, eficiencia en el control de arvenses, lo cual favoreció el crecimiento de las plantas y maximizó la productividad de las plantas. Los reportes de Pereira et al. (2001), Cavalieri et al. (2008) y Biffe et al. (2010) determinaron que los herbicidas diuron, metolaclor, atrazina, metrubizin, ametrina e isoxaflutole pueden mostrar selectividad y no generar reducciones en la productividad de las plantas de yuca, siendo utilizados como herramientas de control de arvenses en yuca.

\section{CONCLUSIONES}

Se encontró tolerancia a herbicidas para la variedad de yuca MCOL-2215, por lo cual pueden ser incluidos como herramientas de manejo de arvenses en el cultivo de yuca en el departamento de Bolívar (Colombia). La mezcla de herbicidas metolacloro + atrazina fue la más eficiente en el control de arvenses y selectiva al cultivo de yuca en época de preemergencia. La aplicación combinada de estos herbicidas incrementó en 4,34 $\mathrm{tha}^{-1}$ los rendimientos del cultivo en comparación con el método tradicional. La combinación de métodos de control (manual y químico) en sistemas de cultivo de yuca permite optimizar los rendimientos e incrementar la rentabilidad del cultivo.

\section{Agradecimientos}

Esta investigación tuvo el apoyo financiero del Ministerio de Agricultura y Desarrollo Rural quien otorgo los recursos a través de convenio TV-16 (C08592). La Corporación Colombiana de Investigación Agropecuaria (Corpoica) sede Carmen de Bolívar suministró el apoyo logístico necesario para su desarrollo. CORPOICA cambio su identidad legal a AGROSAVIA a partir de junio de 2018.

Conflicto de intereses: el manuscrito fue preparado y revisado con la participación de los autores, quienes declaran no tener algún conflicto de interés que coloquen en riesgo la validez de los resultados aquí presentados. Así mismo declaramos no tener conflictos con las empresas distribuidoras de agroquímicos.

\section{REFERENCIAS BIBLIOGRÁFICAS}

Agronet. 2016. Estadísticas de área cosechada, producción y rendimiento de yuca, 2000-2016. En: http://www. agronet.gov.co; consulta: enero de 2018.

Aguilera, M. 2012. La yuca en el Caribe colombiano. De cultivo ancestral a agroindustrial. Economía Regional 158. Banco de la República, Bogotá.

Alemán, F. 2004. Manejo de arvenses en el Trópico. 2a ed. Universidad Nacional Agraria. Managua, Nicaragua.

Alves, E., C. da Conceição de Matos, E. Alves, D. Valadão Silva, J. Barbosa dos Santos, G.A Mendes y C. Teixeira da Silva. 2015. Respostas fisiológicas da mandioca à aplicação de herbicidas. Ciênc. Agrár. 36(2), 645-656. Doi: 10.5433/1679-0359.2015v36n2p645.

Aramendiz-Tatis, H., C. Cardona-Ayala y R. De Oro. 2010. Periodo de interferencia de arvenses en el cultivo de berenjena (Solanum melongena L.). Agron. Colomb. 28(1), 81-88.

Arrieta, J.M., A. Martínez, J.L. Romero, A. Contreras, L. Bracho y G. Gamero. 2004. Principales arvenses del asocio yuca/maíz en la región Caribe colombiano. Editorial Siglo XXI, Bogotá.

Biffe, D.F., J. Constantin, J.R. Oliveira, F.A. Ríos, L.H.M. Franchini y A. Gemelli. 2010. Avaliação de herbicidas para dois cultivares de mandioca. Planta Daninha 28(4), 807-816.

Cavalieri, S.D., J.R.S. Oliveira, J. Constantin, D.F. Biffe, F.A. Rios y L.H.M. Franchini. 2008. Tolerância de híbridos de milho ao herbicida nicosulfuron. Planta Daninha 26(1), 203-214. Doi: 10.1590/S0100-83582008000100021

Costa, N.V., G.C. Pavan, R.F. Douradoda, A.C.P.R. Costa y E.S. Vasconcelos. 2013. Seletividade de herbicidas aplicados com óleo mineral na cultura da mandioca 'Cascuda'. Rev. Bra. Herb. 12(3), 251-259. Doi: 10.7824/ rbh.v12i3.204

Da Costa, N.V., M.M. Arrúa, D.A. Sontag, D.C. De Andrade y J.B.D. Junior. 2014. Seletividade de herbicidas residuais e da mistura com glyphosate aplicados após a poda da mandioca 'Fécula Branca'. Rev. Bras. Herb. 13(1), 23-30. Doi: 10.7824/rbh.v13i1.263

Enyong, J.F.K., N.U. Ndaeyo, B.A. Ndon, L.A. Ugbe y E.A. Akpan. 2013. Preliminary evaluation of effects of herbicide types and rates on growth and yield of cassava (Manihot esculenta Crantz). Int. J. Basic Appl. Sci. 2(2), 65-70.

FAO. 2016. Cassava production statistics. En: Faostat, http://www.fao.org/faostat/es/\#data/OC; consulta: enero de 2018. 
Franciscon, H., N.V. Da Costa, A.C.P.R. Da Costa, S.D. Ferreira, G. Moratelli, A.C. Salvalaggi y M.A.M. Arrúa. 2016. Eficacia y selectividad de mezclas de herbicidas en el cultivo de mandioca. Rev. Fac. Agron. 115(2), 209-219.

Guevara, J., C. Molina, L. Santana y H. Rodríguez. 2004. Estudio general de suelos y zonificación de tierras departamento de Bolívar. 2a ed. Instituto Geográfico Agustín Codazzi (IGAC), Bogotá.

Gutiérrez, W., J. Morán, B. Daboin, J. Ferrer, B. Medina e Y. Villalobos. 2008. Evaluación de diferentes herbicidas sobre el control de arvenses, desarrollo, rendimiento y beneficio. Rev. Fac. Agron. 25(1), 26-42.

Johanns, O. y R.L. Contiero. 2006. Efeitos de diferentes períodos de controle e convivência de plantas daninhas com a cultura da mandioca. Rev. Cien. Agron. 37(3), 326-331.

Lugo-Torres, M.D.L. y M. Díaz. 2007. Weed control in sweet potato (Ipomoea batatas L.). J. Agric. Univ. Puerto Rico 91(3-4), 161-167.

Matheus, J., G. Romay y M.A. Santana. 2004. Efecto de tres herbicidas pre-emergentes en el establecimiento en campo de plantas in vitro de yuca (Manihot esculenta Crantz). Act. Científ. Venez. 55, 191-197.

Moura, G.M. 2000. Interferência de plantas daninhas na cultura de mandioca (Manihot esculenta) no estado do Acre. Planta Daninha 18(3), 451-456. Doi: 10.1590/ S0100-83582000000300009

Nduwumuremyi, A., R. Melis, P. Shanahan y A. Theodore. 2017. Interaction of genotype and environment effects on important traits of cassava (Manihot esculenta Crantz). Crop. J. 5(5), 373-386. Doi: 10.1016/j. cj.2017.02.004.

Nedunchezhiyan, M., C.S. Ravindran y R. Velumani. 2013. Weed management in root and tuber crops in india: critical analysis. J. Root Crops. 39(2), 13-20.

Nedunchezhiyan, M., G. Byju, S.S. Veena y V. Ravi. 2017. Herbicides and polythene mulching effects on yield of cassava. Indian. J. Weed. Sci. 49(1), 58-62. Doi: 10.5958/0974-8164.2017.00014.4

Olorunmaiye, P.M., S.T.O. Lagoke, J.A. Adigun y O.R. Orija. 2013. Effect of intercropping with maize on weed diversity in cassava. Environ. Exp. Biol. 11(1),189-193.

Owusu-Darko, P.G., A. Paterson y E.L. Omenyo. 2014. Cocoyam (corms and cormels) dan underexploited food and feed resource. J. Agric. Chem. Environ. 3(1), 2229. Doi: $10.4236 /$ jacen.2014.31004.

Pereira, F.D.A.R., M.D. Oliveira, R. Bazoni, C.A.C. Busato, L.C. Goes y A.G.D. Rocha. 2001. Estudo da seletividade de herbicidas em cultivares de milho (Zea mays L.), nas condições ambientais de Mato Grosso do Sul. Ens. Ciênc. 5(2), 119-140.
Pérez, J.V., L.L. Luna y O. Burbano. 2018. Acumulación de biomasa en yuca (Manihot esculenta C.) ante la respuesta a la aplicación de fertilizantes NPK y biofertilizantes en condiciones de invernadero en dos tipos de suelo. Rev. Colomb. Cienc. Hortic. 12(2), 456-463. Doi: 10.17584/rcch.2018v12i2.7676.

Reddy, P.P. 2015. Plant protection in tropical root and tuber crops. Springer India, New Delhi. Doi: 10.1007/978-81-322-2389-4.

Salau, A.W., F.O. Olasantan, J.G. Bodunde y E.A. Makinde. 2015. Soil temperature and moisture content changes with growth and yield of cassava-vegetable intercrops. Arch. Agron. Soil Sci. 61 (4), 447-460. Doi: 10.1080/03650340.2014.939958.

Scariot, C.A., N. Vilanova, E.P. Bosquese, D.C. de Andrade y D.A. Sontag. 2013. Seletividade e eficiência de herbicidas aplicados em pré-emergência na cultura da mandioca. Pesq. Agropec. Trop. 43(3), 300-307.

Silva, A.A., T. Sediyama, J.B. Santos, D.V. Silva, A.C. França y E.A. Ferreira. 2012. Manejo de plantas daninhas na cultura da mandioca. Planta Daninha 30(4), 835-841.

Silva, D.V., J.B. Dos Santos, H.M. Silveira, F.P. De Carvalho, M.D.C. Neto, E.A. Ferreira y P.R. Cecon. 2011. Tolerância de cultivares de mandioca aos herbicidas fomesafen e fluazifop-p-butil. Rev. Brasil. Herb. 10(3), 219-231. Doi: 10.7824/rbh.v10i3.125

Silva, D.V., J.B. Santos, J.P. Cury, F.P. Carvalho, E.B. Silva, J.S.C. Fernandes y G. Concenço. 2013. Competitive capacity of cassava with weeds: implicationson accumulation of dry matter. Afr. J. Agric. Res. 8 (6), 525 531. Doi: $10.5897 /$ ajar12.652

Silva, F.M.L., M.L. de Abreu, E.L. Brachtvogel, F. Curcelli, M.J. Gimenes y A.C. Da Costa Lara. 2010. Moléculas de herbicidas seletivos à cultura da mandioca. Rev. Tróp. Cie. Agra. Biol. 3(2), 61-72.

Silveira, H.M., D.V. Silva, J.B. dos Santos, M.D.C. Neto, E.A. Ferreira, F.P. De Carvalho y T. Sediyama. 2012. Sensibilidade de cultivares de mandioca ao herbicida mesotrione. Rev. Brasil. Herb. 11(1), 24-31.

Streck, N.A., D.G. Pinheiro, Z.A. Junior, L.F. Gabriel, T.S.M. Rocha, A.T.D. Souza y M.R.D. Silva. 2014. Effect of plant spacing on growth, development and yield of cassava in subtropical environment. Bragantia 73(4), 407-415. Doi: 10.1590/1678-4499.0159.

Varanasi, A., P.V. Prasad y M. Jugulam. 2016. Impact of climate change factors on weeds and herbicide efficacy. Adv. Agron. 135, 107-146. Doi: 10.1016/ bs.agron.2015.09.002

Vencill, W.K., R.L. Nichols, T.M. Webster y J.K. Soteres. 2012. Herbicide resistance: toward an understanding of resistance development and the impact of herbicide-resistant crops. Weed Sci. 60(1), 2-30. Doi: 10.1614/ WS-D-11-00206.1 
Villordon, A.O., I. Ginzberg y N. Firon. 2014. Root architecture and root and tuber crop productivity. Trends Plant Sci. 19(7), 419-425. Doi: 10.1016/j.tplants.2014.02.002

Weerarathne, L.V.Y., B. Marambe y B.S. Chauhan. 2017. Does intercropping play a role in alleviating weeds in cassava as a non-chemical tool of weed management?
A review. Crop Prote. 95(1), 81-88. Doi: 10.1016/j. cropro.2016.08.028.

Zimdahl, R.L. 2018. Methods of weed management. pp. 272-281. En: Zimdahl, R.L. (ed.). Fundamentals of weed science. $5 \mathrm{a}$ ed. Elsevier-Academic Press, London. Doi: 10.1016/B978-0-12-811143-7.00010-X 